\title{
Bacteriology of blood stream infections in diabetic and non diabetic patients under the course of hemodialysis: a mini-review
}

Keywords: diabetic, non-diabetic, Staphylococcus aureus, CoNS, hemodialysis(HD)

\section{Introduction}

In general, infectious diseases are more frequent and serious in patients with diabetes mellitus, which potentially increases their morbidity or mortality. Patients with diabetes may experience higher risk of Staphylococcus aureus bacteremia (SAB) than patients without diabetes due to decreased immunity or coexisting morbidities. ${ }^{1}$

Patients with ESRD (End-stage renal disease) have a high incidence of bacterial infections. ${ }^{2}$ This increased susceptibility to bacterial infections is due in part to the acquired immune deficiency state of uremia, advanced age, and comorbid conditions such as diabetes mellitus (DM), as well as the frequent and repetitive exposure of patients to potential infectious risk factors during the normal course of dialysis therapy. ${ }^{3}$

Bloodstream infection was more frequent in diabetics than in nondiabetics (25.8/1000 admissions vs. 5.8/1000 admissions, $\mathrm{p}<0.0001)$. Diabetics had a 4.4-fold higher risk of bloodstream infection, were more prone to sepsis of unknown origin and had more septic complications than non-diabetics. The mortality rate was similar in the two groups. Urinary tract infection was the predominant source, and Escherichia coli the most frequent microorganism in both groups. Klebsiella pneumoniae was more frequent in diabetics than in nondiabetics $(18 \%$ vs $5 \%, \mathrm{p}<0.001){ }^{4}$ Staphylococci were a common cause of bacteremic infections of the extremities in diabetic patients (12 of $19,63 \%)$ and nondiabetic patients (20 of $50,40 \%){ }^{2}$

A cross-sectional study was conducted for a period of six months in National Kidney Center of Nepal to determine the prevalence of bacteremia in diabetic and non-diabetic patients undergoing hemodialysis course.Blood samples were collected and inoculated in brain heart infusion biphasic media in blood culture bottle and incubated aerobically at $37^{\circ} \mathrm{C}$ for $18-24$ hours. Macroscopic and microscopic observations and conventional biochemical tests were done to identify the isolated organisms. Data were analysed using Statistical Package for the Social Sciences version 17.

\section{Results}

Table 1 \& Table 2

Table I Growth of organisms in diabetic and non- diabetic patients

\begin{tabular}{lllll}
\hline & \multicolumn{2}{l}{ Diabetic mellitus } & Total (\%) & $\begin{array}{l}\text { p- } \\
\text { value }\end{array}$ \\
\cline { 2 - 4 } & Diabetic (\%) & Non-diabetic (\%) & & \\
\hline Growth & $2(7.7)$ & $24(92.3)$ & $26(26)$ & 0.35 \\
No growth & $11(14.9)$ & $63(85.1)$ & $74(74)$ & \\
Total & $13(13)$ & $87(87)$ & $100(100)$ & \\
\hline
\end{tabular}

Volume 7 Issue I - 2019

\author{
Prasansah Shrestha, Nabaraj Pokhrel, Anil \\ Dev Pant \\ Department of Microbiology, Tribhuvan University, Nepal
}

Correspondence: Shrestha Prasansah, Department of Microbiology, National College (Tribhuvan University), Kathmandu, Nepal, Email praisehonour@gmail.com

Received: July 07, 2016 | Published: January 31, 2019

Table 2 Microorganisms isolated from blood culture

\begin{tabular}{lll}
\hline Microorganisms & Diabetic $(\mathbf{n}=\mathbf{2})$ & Non- diabetic $(\mathbf{n}=\mathbf{2 4})$ \\
\hline S. aureus & 2 & $\mathrm{I} 4$ \\
CONs & - & 7 \\
E.Coli & - & $\mathrm{I}$ \\
Pseudomonas aeruginosa & - & $\mathrm{I}$ \\
Klebsiella pneumoniae & - & $\mathrm{I}$ \\
Total & 2 & 24 \\
\hline
\end{tabular}

\section{Conclusion}

In conclusion, there was no association in between bacteremia and diabetes. The diabetics are at high risk of blood stream infection. ${ }^{3}$ A bloodstream infection was detected significantly in asymptomatic patients. The predominant organism found to be $S$. aureus.

\section{Acknowledgments}

None.

\section{Conflicts of interest}

Authors declare that there is no conflict of interest.

\section{References}

1. Jesper S, Mette S, Henrik CS, et al. Diabetes and risk of communityacquired Staphylococcus aureus bacteremia: A population-based case control study. Eur J Endocrinol. 2016;174(5):631-639.

2. Leibovici L, Samra Z, Konisberger H, et al. Bacteremia in adult diabetic patients. Diabetes Care. 1991;14(2):89-94.

3. Stoecklea M, Kaechb C, Trampuzb A, et al. The role of diabetes mellitus in patients with bloodstream infections. Swiss Med Wkly. 2008;138(3536):512-519.

4. Sarnak MJ, Jaber BL. Mortality caused by sepsis in patients with endstage renal disease compared with the general population. Kidney Int. 2000;58(4):1758-1764. 